\title{
Evidential Identification of New Target based on Residual
}

\author{
L. Zheng, Z. Zhang, Y. Deng
}

\author{
Lei Zheng, Zhiguo Zhang \\ College of Infomation Science and Technology \\ Jinan University, Guangzhou, China \\ LeiZhen@@stu2015.jnu.edu.cn, zhangzhiguo@stu2015.jnu.edu.cn
}

\author{
Yong Deng* \\ 1. Big Data Decision Institute, \\ Jinan University \\ Tianhe, Guangzhou 510632, China \\ 2. Institute of Fundamental and Frontier Science, \\ University of Electronic Science and Technology of China \\ Chengdu, 610054, China \\ *Corresponding author: prof.deng@hotmail.com
}

\begin{abstract}
Both incompleteness of frame of discernment and interference of data will lead to conflict evidence and wrong fusion. However how to identify new target that is out of frame of discernment is important but difficult when it is possible that data are interfered. In this paper, evidential identification based on residual is proposed to identify new target that is out of frame of discernment when it is possible that data are interfered. Through finding the numerical relation in different attributes, regress equations are established among various attributes in frame of discernment. And then collected data will be adjusted according to three mean value. Finally according to weighted residual it is able to decide whether the target requested to identify is new target. Numerical examples are used to verify this method.

Keywords: evidence theory, identification of new target, linear regression, residual.
\end{abstract}

\section{Introduction}

How to deal with uncertainty in real life is still an open issue $[13,29,30,54,62,66,76]$. Many math tools, such as fuzzy set $[3,25,58,64,67,71,73]$, rough sets $[19,21,36,40,41,52]$, entropy function $[1,2,49,49,70]$ and $\mathrm{D}$ numbers $[4,8,33,39,75]$, are presented to address this issue $[14,16,37,65,71,74]$. Among these efficient tools, Dempster Shafer evidence theory [5, 44] has been paid greatly attention recently. Since Dempster-Shafer theory (DS theory) is proposed $[5,44]$, it has been widely used in information fusion $[9,28,32,51,60,61]$, control systems $[10,31]$, uncertainty modelling $[7,19,38,46,47,59]$ decision making $[26,27,35,73]$, risk and reliability analysis $[15,22,56,63,74]$ and other fields $[6,50,68,69]$. In DS theory , a basic probability assignment(BPA) is distributed to power sets of the frame of discernment and the sum of BPA is always one when supposed that elements in the frame of discernment are mutually exclusive and exhaustive. However, in fact, the frame of discernment that we assume or have known is not complete, that is to say probably there are some unknown species in a world, which is called an open world $[20,45]$.It should be mentioned that an open issue in evidence theory is the conflict management $[48,53,55,72]$, which is also partially caused by open world.

And in past decades, a large amount of research has been conducted on this issue. Generalized evidential theory (GET) is proposed [11], which defines a novel concept called generalized basic probability assignment (GBPA) to model uncertain information, and provides a generalized combination rule (GCR) for the combination of GBPAs, and builds a generalized conflict model to measure conflict among evidences in an open world. Not only that, in [23], the conflict is 
explored in a closed world and the result of evidence fusion is able to converge to correct answer when using the proposed method.

In addition, the identification of frame of discernment also is an open problem. Allocating a non-null value to the mass function on the empty is able to express and judge the incompleteness of frame of discernment in certain condition. As considering the case of potentially heterogeneous sources, Johan Schubert proposed a novel way to construct and evaluate alternative frames of discernment [43]. And dynamic estimation of the discernment frame in belief function theory is proposed by Wafa Rekik et al. [42] which using Cartesian product whose axes correspond to elementary discernment frames dealing with the relevance of each potential hypothesis to judge and update the frame of discernment.

However all of these works assume the collected data is correct and they are not interfered or just are interfered a little. In other words, only if the collected data are not interfered or just are interfered a little, the incompleteness of frame of discernment will be identified. In fact the collected data is very probably interfered. And the known species will be recognized as new target using existing method when the collected data is interfered. Therefore considering incompleteness identification under data interference condition have utilitarian value.

In this paper, evidential identification based on residual is proposed to identify incompleteness of frame of discernment. First assume that the frame of discernment is complete and there is a closed world. Then the method proposed in [11] is used to fuse data, which can get correct result even in evidence conflict in an closed world. Secondly through finding the numerical relation in different attributes, regress equations are established for different species and residual vector is calculated. The third step is that adjust collected data according to offset degree. The forth step is to plug the adjusted data into regress equation to calculate residual vector and weighted residual. Finally new target or perturbation of data is identified according to weighted residual. Numerical examples are used to introduce this method.

The rest of the paper is organized as follows. In Section 2, Dempster-Shafer theory, generalized evidence theory and evidence distance are briefly introduced. In Section 3, a new evidential identification based on residual is proposed. In section4 numerical examples are used to illustrate the behaviour of the new evidential identification based on residual. Section 5 concludes the main contribution of the paper.

\section{Preliminaries}

In this section, some preliminaries are briefly introduced below.

\subsection{Dempster-Shafer theory of evidence [5, 44]}

In Dempster-shafer theory, basic probability is distributed to power sets of the frame of discernment whose elements are mutually exclusive and exhaustive. Some terminology and notions are defined below to explain theory better.

Let $\Theta$ be a set of $N$ mutually exclusive and exhaustive elements, which means the problem has $N$ possible values. The following set is called the frame of discernment.

$$
\Theta=\left\{H_{1}, H_{2}, \ldots, H_{N}\right\} .
$$

$P(\theta)$ is the power set composed of $2^{N}$ elements A of $\theta$, representing the object is in A.

$$
\begin{aligned}
P(\Theta)= & \left\{\phi, H_{1}, \cdots, H_{N},\left(H_{1}, H_{2}\right),\left(H_{1}, H_{3}\right), \ldots,\left(H_{N-1}, H_{N}\right), \cdots,\right. \\
& \left.\left(H_{1}, H_{2}, H_{3}\right), \cdots, \Theta\right\} .
\end{aligned}
$$


A basic probability assignment (BPA) is a function from $P(\theta)$ to $[0,1]$ defined by:

$$
m: \quad P(\Theta) \rightarrow[0,1]
$$

and which satisfies the following conditions:

$$
\begin{aligned}
& \sum_{A \in P(\Theta)} m(A)=1, \\
& m(\phi)=0 .
\end{aligned}
$$

where $m(A)$ represents the belief to $A$.

\subsection{Generalized evidence theory}

Generalized evidence theory is proposed by Deng [11], which generalize DS theory of evidence. When the frame of discernment is probably incomplete, generalized evidence theory is used to replace classical evidence theory, which defines a novel concept called generalized basic probability assignment (GBPA) to model uncertain information, and provides a generalized combination rule (GCR) for the combination of GBPAs, and builds a generalized conflict model to measure conflict among evidences in an open world [11]. But if the frame of discernment is complete, generalized evidence theory degrades into classical evidence theory.Some terminology and notions are defined below to explain theory better.

Let $U$ be a frame of discernment in an open world, Which consists of $N$ mutually exclusive elements.

$$
U=\left\{H_{1}, H_{2}, \ldots, H_{N}\right\}
$$

And its power set $2_{G}^{U}$ is consisted of $2^{U}$ propositions, which contains empty set. For $\forall A \in U$, If the function $m: 2_{G}^{U} \rightarrow[0,1]$ satisfies

$$
\sum_{A \in U} m_{G}(A)=1
$$

the function $m$ is called Generalized Basic Probability Assignment(GBPA). In this paper $m(\Phi)$ is not restricted to zero, which means the basic probability assignment to the proposition out of discernment. In other words, $m(\phi)$ represents the probability that the target is out of discernment.

\subsection{Existing evidence distance}

To measure the distance between two bodies of evidence, Jousselme defined a function from vector made up of BPAs to real number [24]. Let $m_{1}$ and $m_{2}$ be two BPAs on the same frame

of discernment $\Theta$, containing $\mathrm{N}$ mutually exclusive and exhaustive hypotheses. The distance between $m_{1}$ and $m_{2}$ is:

$$
d_{B P A}\left(m_{1}, m_{2}\right)=\sqrt{\frac{1}{2}\left(\overrightarrow{m_{1}}-\overrightarrow{m_{2}}\right)^{T} \underline{\underline{D}}\left(\overrightarrow{m_{1}}-\overrightarrow{m_{2}}\right)},
$$

where $\overrightarrow{m_{1}}$ and $\overrightarrow{m_{2}}$ are the associated vectors of BPAs $m_{1}$ and $m_{2}$ and $\underline{\underline{D}}$ is a $2^{N} \times 2^{N}$ matrix whose elements are

$$
\begin{aligned}
& D(A, B)=\frac{|A \cap B|}{|A \cup B|}, \\
& A, B \in P(\Theta) .
\end{aligned}
$$




\section{The proposed evidential identification of new target based on residual}

In this section evidential identification of new target based on residual is proposed. Like many ways of identifying the frame of discernment, at first assume the frame of discernment is compete and the world is closed. And then based on conflicts management in close world in [11], BPAs are produced and fusion result is obtained. Secondly through finding the numerical relation in different attributes, regress equations are established for different species and residual vector is calculated. The third step is that adjust collected data according to offset degree. The forth step is to plug the adjusted data into regress equation to calculate residual vector and weighted residual. Finally new target or perturbation of data is identified according to weighted residual. And the flow diagram is shown in Figure 1.

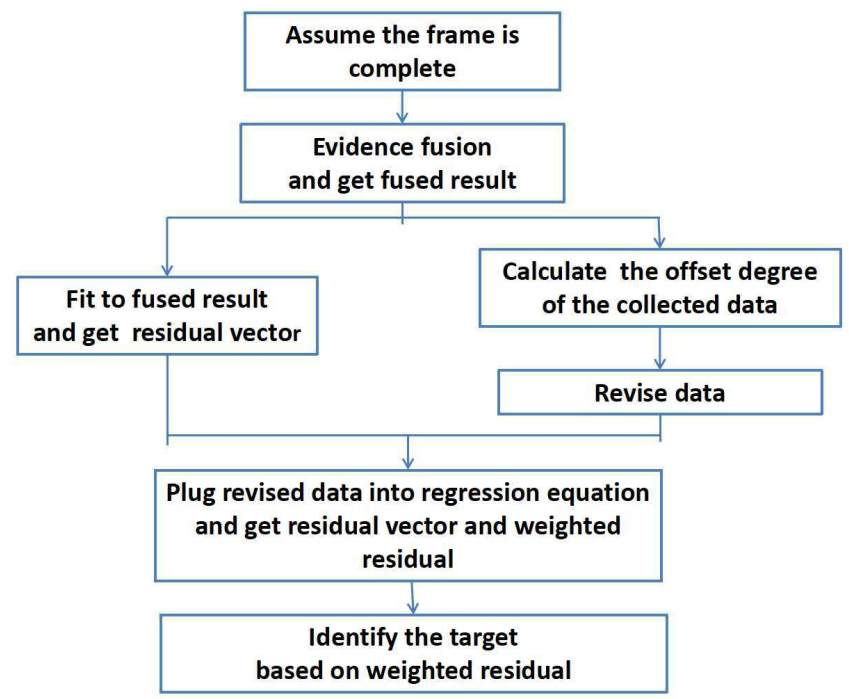

Figure 1: The flow diagram of new method

\subsection{Evidence fusion}

First suppose the frame of discernment is complete, conflicts management in close world. The method proposed in [11] is used to produce and combine BPAs. The reason why this method is chose is that this method can converge to correct answer even in strongly conflict condition when the frame of discernment is complete.

Furthermore when supposing that the frame of discernment is complete, many methods can be used to produce BPAs and fuse BPAs, which will converge to correct target and show a great result.

\subsection{Linear regression}

In most cases, there is strong correlation among different attributes in the same species. Furthermore the correlation changes with species. For example, the relation expressions for heights and weights change with sex. Therefore the relation expression for various attributes of different species is a good tool to distinguish species. In this paper, relation expression will be established among different attributes for fused result. And it is well known that there are 
many ways to represent the correlation for different attributes, such as covariance matrix, linear regression, nonlinear regression.

For simplifying linear regression is elected to represent the correlation among attributes which is able to take every attribute into account at the same time. In other words, the following equation is used to represent one species.

$$
a_{1} x_{1}+a_{2} x_{2}+\cdots+a_{n} x_{n}=a
$$

Where $x_{i}$ represents the value of ith attribute in certain species, $a_{i}$ represents the weight of ith attribute or is called ith regression coefficient in relation expression which can be calculated by least square method and $a$ is arbitrary constant which is an artificial constant. In addition, the value of a just affects regression coefficient increasing or decreasing manifold at the same time.

In the process of linear regression, residual vector can be calculated. According to the vector, histogram and probability contribution function for residual are obtained.

Example 1. Take Iris data (http://archive.ics.uci.edu/ml/datasets/Iris) as an example. Suppose that Setosa is one of elements in frame of discernment and twenty pieces of sample data are known. There are four attributes in one sample which are sepal length, sepal width, petal length and petal width. The chosen data are shown in Figure 2

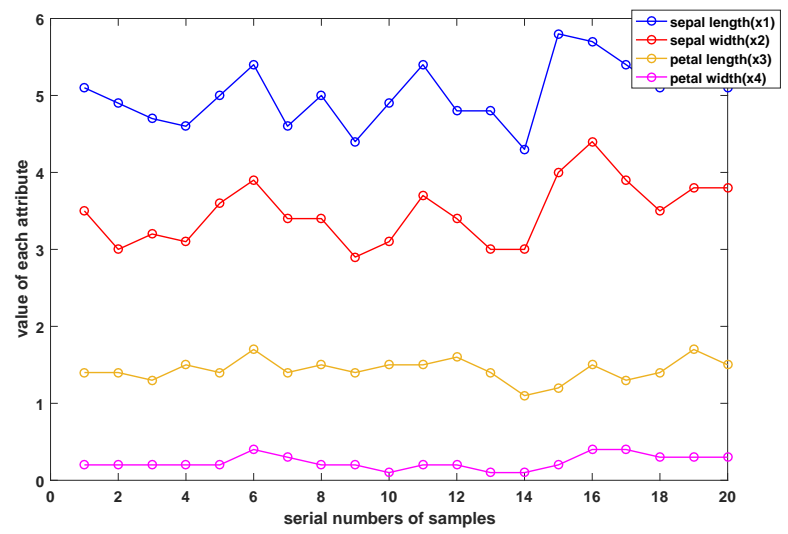

Figure 2: Twenty pieces of sample data which are used in liner regression

Let $X_{1}$ is the vector of value of sepal length, $X_{2}$ is the vector of value of sepal width, $X_{3}$ is the vector of value of petal length and $X_{4}$ is the vector of value of petal width, constant $a$ is 100. Using least square method, the value of $a_{1}, a_{2}, a_{3}, a_{4}$ is calculated and linear regression of Setosa is obtained.

$$
10.98 x_{1}+4.827 x_{2}+26.98 x_{3}-47.89 x_{4}=100 .
$$

If setting constant $a$ is 1000 the linear regression of Setosa is

$$
109.8 x_{1}+48.27 x_{2}+169.8 x_{3}-478.9 x_{n}=1000 .
$$

The regression coefficient of the second equation is just ten times of the first equation when the second constant $a$ is ten times of the first constant. And in the process of linear regression, residual vector can be calculated. And histogram and probability contribution function for residual are shown in Figure 3. 

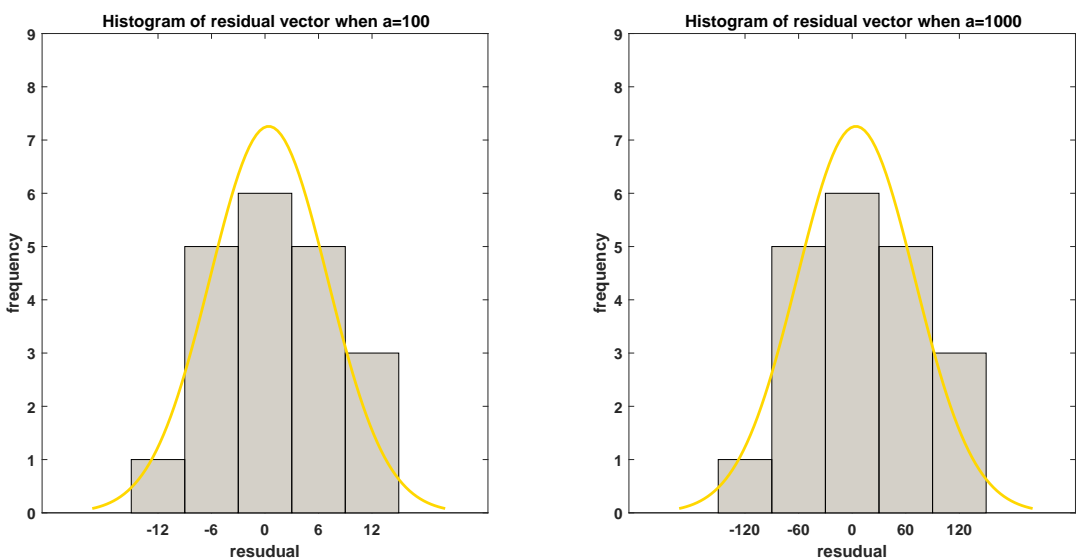

Figure 3: Histogram of residual vector when $\mathrm{a}=100$ and $\mathrm{a}=1000$

\subsection{Data processing}

If the target requested to identify is one of the elements of frame discernment and the data are not disturbed, the collected data will satisfy Eq.(9) equation or the residual will be small. Not only that, when revising noisy data and take it into Eq.(9), the revised data will satisfy Eq.(9) or the residual will be small. At the same time, if the target requested to identify is out of frame, after the same data revising, the revised data will not satisfy Eq.(9) or the residual will be large.

But how to revise data to satisfy these conditions mentioned above?

First offset degree is defined as follows. Let frame of discernment

$$
\theta=\left\{H_{1}, H_{2}, \cdots, H_{n}\right\},
$$

the target which is requested to identify is $S . S_{j}$ represents jth attribute for $S$, and $H_{i j}$ represents jth attribute for element $H_{i}$.

$$
\begin{aligned}
t_{j} & =\frac{\text { threemean }\left(S_{j}\right)}{\text { threemean }\left(H_{i j}\right)} \\
\operatorname{threemean}(A) & =\frac{1}{4} Q_{1}+\frac{1}{2} M+\frac{1}{4} Q_{3}
\end{aligned}
$$

where $t_{j}$ is defined as jth attribute offset degree for target requested to identify $S$. $A$ is a vector, $Q_{1}$ is upper quartile for vector $A, M$ is mean for vector $A$, and $Q_{3}$ is lower quartile for vector $A$.

Because threemean have disturbance rejection for extremum and abnormal data have no effect on offset degree, it is appropriate to use threemean to calculate offset degree.

And then revised data is defined as follows.

$$
\widehat{S_{j}}=\frac{1}{t_{j}} \times S_{j}
$$

Because when taking the fusion method in [11], if the target is one of element in frame of discernment, it can diverge to correct solution. It is enough to calculate offset degree between target requested to identify and fused result and revise collected data according to fused result. It is no need to calculate offset degree between target requested to identify and every elements in frame of discernment.

Next plug these revised data into linear regression Eq.(9) and obtain residual $r$.

$$
r=a-\left(a_{1} H_{1}+a_{2} H_{2}+\cdots+a_{n} H_{n}\right)
$$




\subsection{Residual vector processing}

Let $R$ represent residual vector of collected data of target requested to identify, and the ith element of $R, R(i)$, represents the residual between the ith collected sample data and linear regression of fusion result calculated in (9). If there is extremum in collected data, the part of component of vector will be large. To avoid the effect of extremum, weighted residual is chose. In general condition, the more similar the samples data are, the more confidence will be given, the same as the process of weighted fusion. For simplifying, the weight proposed in [12] consist of evidence distance serves as weight to produce weighted residual.

As we know the less weighted residual is, the more probably the target requested to identify is the convergence. When setting $\alpha$ as rejection probability, the section that locate at both ends in probability density function will be rejected as well as Figure 4.

Therefore according to given rejection probability, residual probability density function or

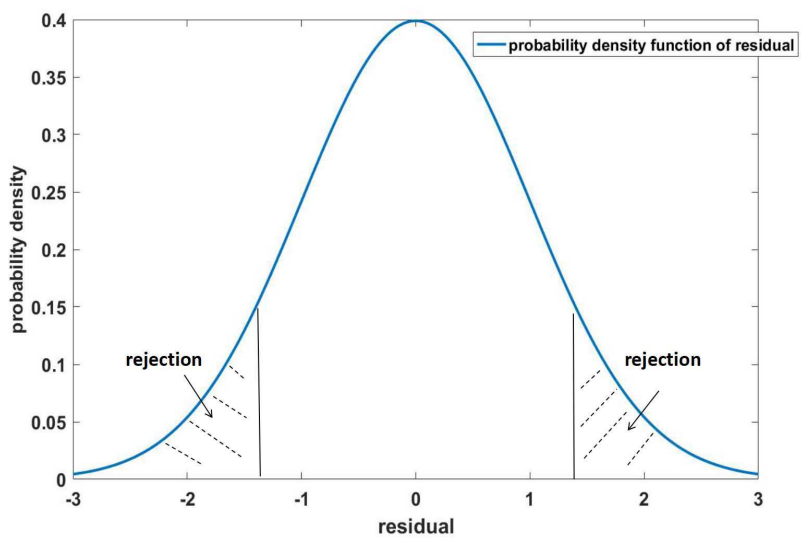

Figure 4: Rejection method according to probability density function

diagram and weighted residual, whether the target requested to identify is a new target is able to identify.

\section{Numerical example}

Real world exist many uncertainty $[17,34,57]$. In this section numerical examples are used to illustrate the validity of evidential identification of new target based on residual.

Take Iris data (http://archive.ics.uci.edu/ml/datasets/Iris) as an example. There are 150 samples in Iris data and each sample has four attributes such as sepal length, sepal width, petal length and petal width. In addition, 150 samples are divided into three classes which are Setosa, Versicolour and Virginica and there are 50 samples in each class.

Let frame of discernment consist of Setosa and Virginica $\theta=\{$ Setosa, Virginica $\}$, and Versicolour is out of frame. To certify the practical of this method, two different conditions are considered. First condition is that the target requested to identify is Versicolour which is out of frame. Second condition is that the target requested to identify is Virginica whose collected data are interfered.

first condition, select 10 samples in Versicolour at random as collected data

Step 1. evidence fusion 
According to method proposed in [11] and collected data, 40 pieces BPA are reduced shown in Figure 5 and evidence distance is obtained.
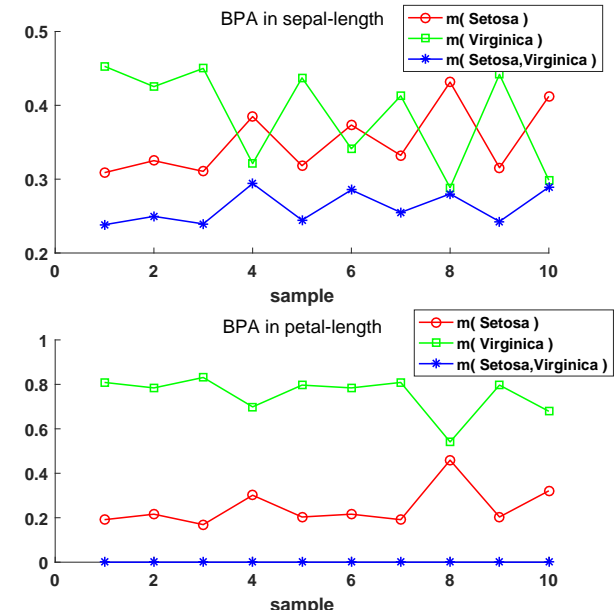
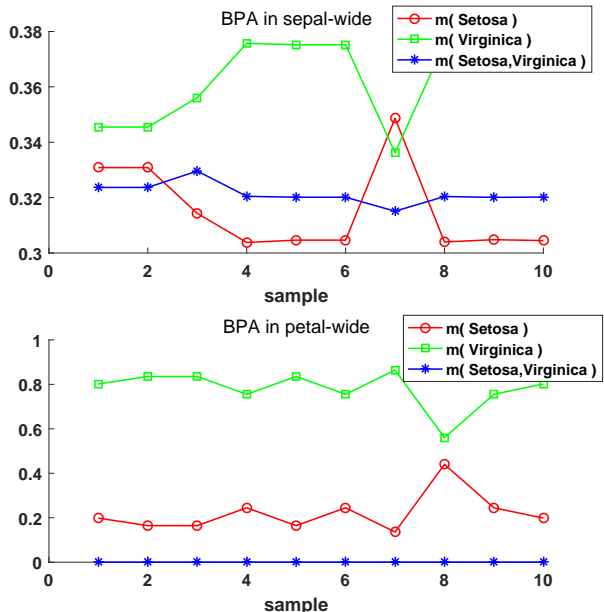

Figure 5: BPAs of collected data

And then fuse these BPAs and result converges to Virginica.

Step 2. linear regression

The Eq. (9) is chose to reflect the relation among four attributes of Virginica. where $x_{i}$ represent the value of ith attribute in Virginica and $a_{i}$ represent the weight of ith attribute or is called ith regression coefficient .

Based least square method, parameters in linear regression are calculated and the relational expression is shown below.

$$
5.15 x_{1}+9.25 x_{2}+4.36 x_{3}+6.75 x_{4}=100
$$

At same time the residuals of each sample can be obtained and represented by histogram shown in Figure 6. According to residual vector, the average of absolute residuals is 6.73.

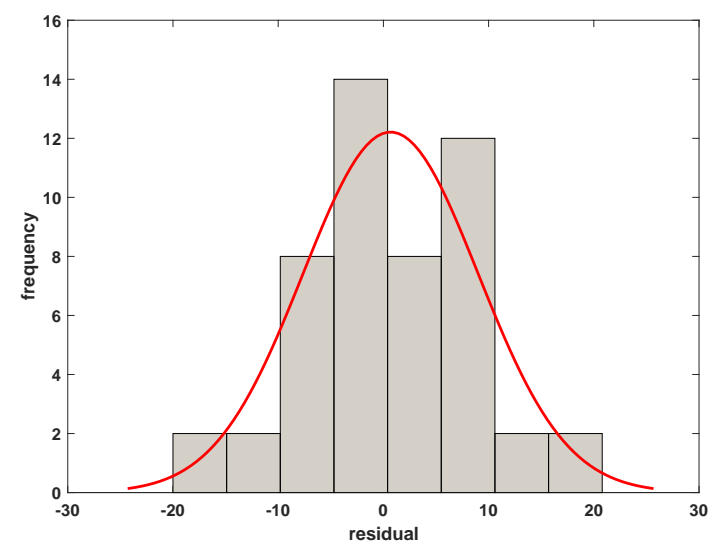

Figure 6: Residual distribution histogram

And when the absolute residual is larger than 7 , there is seventy percent that the target requested to identify is not Virginica. 
Step 3. data processing

Offset degree is calculated as below. Where $S_{i}$ represent the vector of ith attribute for target, $H_{i}$ represent the vector of ith attribute for Virginica.

$$
\begin{aligned}
& t_{1}=\frac{\text { threemean }\left(S_{1}\right)}{\text { threemean }\left(H_{1}\right)}=0.95 \\
& t_{2}=\frac{\text { threemean }\left(S_{2}\right)}{\text { threemean }\left(H_{2}\right)}=0.96 \\
& t_{3}=\frac{\text { threemean }\left(S_{3}\right)}{\text { threemean }\left(H_{3}\right)}=0.81 \\
& t_{4}=\frac{\text { threemean }\left(S_{4}\right)}{\text { threemean }\left(H_{4}\right)}=0.69
\end{aligned}
$$

And then according to offset degree of each attribute, data are revised.

$$
\begin{aligned}
\widehat{S_{i}} & =\frac{1}{t_{i}} \times S_{i}(i=1,2,3,4) \\
\widehat{S} & =\left[\widehat{S_{1}}, \widehat{S_{2}}, \widehat{S_{3}}, \widehat{S_{4}}\right]
\end{aligned}
$$

Step 4. residual vector processing

Take the revised data into linear regression Eq.(16) ten pieces of residual vector can be obtained.The figure of residual vector is shown in Figure 7. And according to [12] the weighted residual 8.3 can be obtained as well.

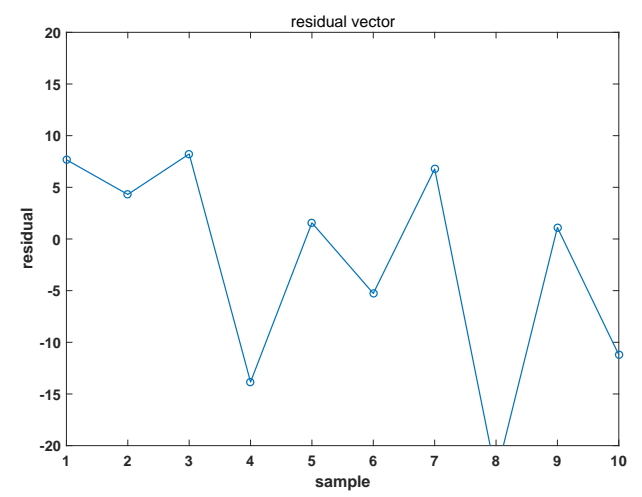

Figure 7: Residual vector of collected data

Because 8.3 is larger than 6.73 and 7 , the probability that target requested to identify is Virginica is not larger than seventy percent. Therefore based on the collected data, target requested to identify is regarded as a new species.

Above all, this method can identify new target.

In the second condition, choose 10 samples in Virginica at random and the sample data becomes 1.25 times.

Step 1. evidence fusion

According to method proposed in [11] and collected data, 40 pieces BPA are reduced shown in Figure 8 and evidence distance is obtained.

And then fuse these BPAs and result converges to Virginica. 

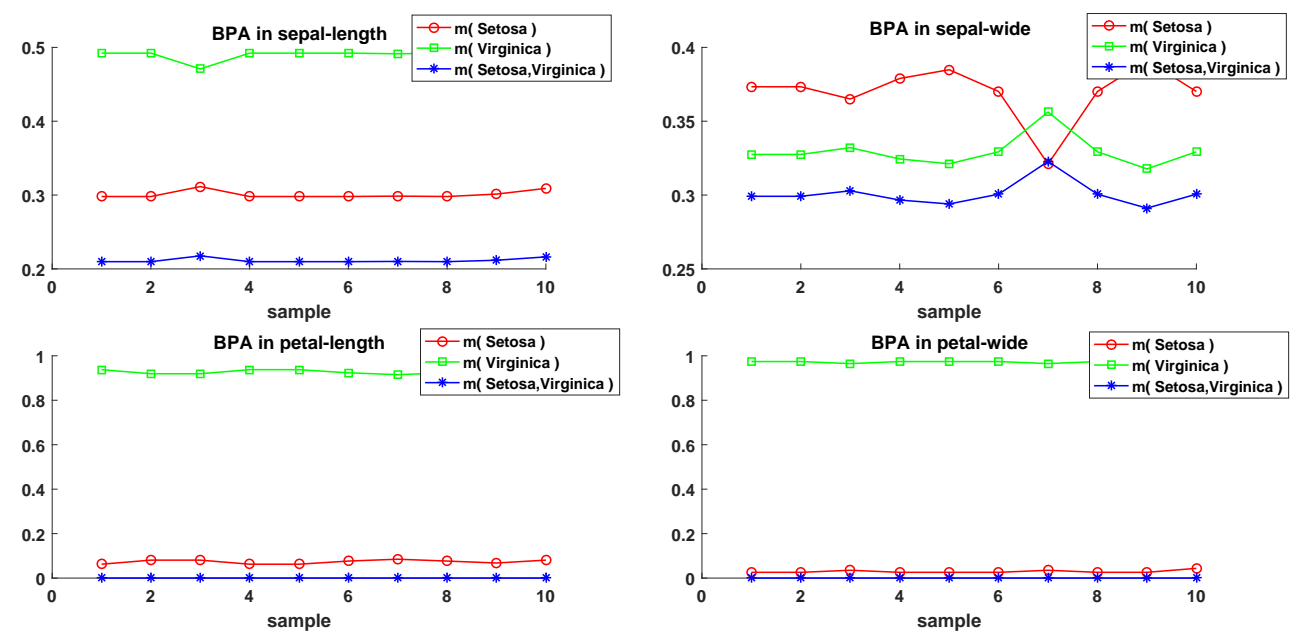

Figure 8: BPAs of collected data

Step 2. linear regression

The following equation is chose to reflect the relation among four attributes of Virginica.

$$
5.15 x_{1}+9.25 x_{2}+4.36 x_{3}+6.75 x_{4}=100
$$

Step 3. data processing

Offset degree is calculated.

$$
t_{1}=1.25, t_{2}=1.28, t_{3}=1.19, t_{4}=1.35
$$

And then according to offset degree of each attribute, data are revised.

Step 4. residual vector processing

Take the revised data into linear regression Eq.(17) ten pieces of residual vector can be obtained.The figure of residual vector is shown in Figure (9). And according to [12] the weighted residual 4.9 can be obtained as well.

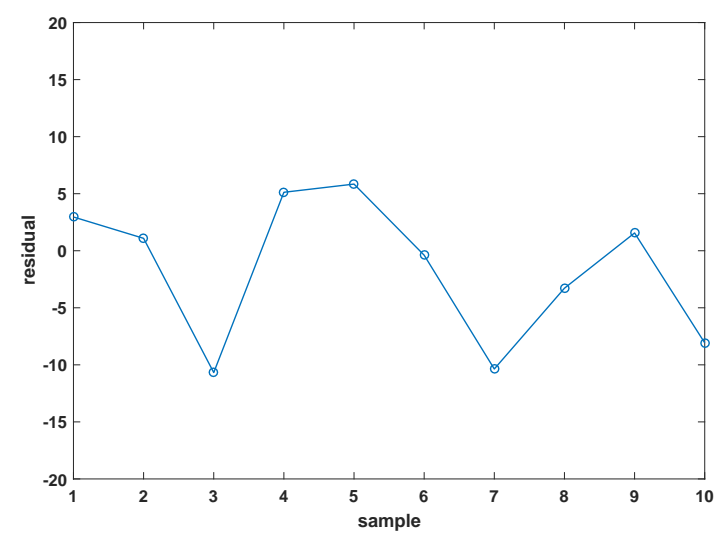

Figure 9: Residual vector of collected data

Because 4.9 is less than 7 and 6.73 , the result that target requested to identify is Virginica is not rejected. 
Above all, when the data are interfered largely, target requested to identify is able to be identified as known species.

\section{Conclusion}

How to identify new target is a significant problem when it probably exists interference. However all of these works assume the collected data is correct and they are not interfered or just interfered a little. In other words, if the collected data are interfered, the identification of new target probably is wrong. The evidential identification of new target based on residual proposed in this paper considers the probability that data are interfered when identifying whether the target is new. And through data fusion, linear regression, data processing and residual vector processing, weighted residual can be obtained which is able to identify whether it is new target. It considers correlation among different attributes. By numerical example, efficiency and practicability of this method are proved. However in this method the setting of rejection probability which affects the accuracy of result is subjective. Therefore how to find a objective way to identify rejection probability will be significant research indicators of further studies. Also for simplifying, liner regression is elected to represent the correlation among attributes, while other method, such as nonlinear regression, will improve the accuracy.

\section{Acknowledgment}

The work is partially supported by National Natural Science Foundation of China (Grant Nos. 61573290, 61503237).

\section{Conflict of interests}

The authors declare that there is no conflict of interests regarding the publication of this paper.

\section{Bibliography}

[1] Abellán, J., Mantas, C. J., Castellano, J. G. (2017): A random forest approach using imprecise probabilities, Knowledge-Based Systems, 134, 72-84, 2017.

[2] Abellán, J. (2017): Analyzing properties of deng entropy in the theory of evidence. Chaos Solitons \& Fractals, 95, 195-199, 2017.

[3] Akyar, H. (2016): Fuzzy risk analysis for a production system based on the nagel point of a triangle. Mathematical Problems in Engineering,2016,(2016-3-31) 2016 (4), 1-9.

[4] Bian, T., Zheng, H., Yin, L., Deng, Y. (2018): Failure mode and effects analysis based on D numbers and topsis. Quality and Reliability Engineering International, Article ID: QRE2268.

[5] Dempster, A. P. (1967): Upper and lower probabilities induced by a multivalued mapping. Annals of Mathematics and Statistics, 38 (2), 325-339, 1967.

[6] Deng, W., Lu, X., Deng, Y. (2018): Evidential Model Validation under Epistemic Uncertainty. Mathematical Problems in Engineering, Article ID 6789635, 2018. 
[7] Deng, X. (2018): Analyzing the monotonicity of belief interval based uncertainty measures in belief function theory, International Journal of Intelligent Systems, Published online, DOI: https://doi.org/10.1002/int.21999, 2018.

[8] Deng, X., Deng, Y. (2018): D-AHP method with different credibility of information, Soft Computing, Published online, doi: 10.1007/s00500-017-2993-9, 2018.

[9] Deng, X., Jiang, W. (2018): Dependence assessment in human reliability analysis using an evidential network approach extended by belief rules and uncertainty measures. Annals of Nuclear Energy, 117, 183-193, 2018.

[10] Deng, X., Jiang, W. (2018): An evidential axiomatic design approach for decision making using the evaluation of belief structure satisfaction to uncertain target values. International Journal of Intelligent Systems, 33 (1), 15-32, 2018.

[11] Deng, Y. (2015): Generalized evidence theory, Applied Intelligence, 43 (3), 530-543, 2015.

[12] Deng, Y., Shi, W. K., Zhu, Z. F., Liu, Q. (2004): Combining belief functions based on distance of evidence, Decision Support Systems, 38 (3), 489-493, 2004.

[13] Dong, Y., Wang, J., Chen, F., Hu, Y., Deng, Y. (2017): Location of facility based on simulated annealing and ZKW algorithms, Mathematical Problems in Engineering, Article ID 4628501, 2017.

[14] Fei, L., Wang, H., Chen, L., Deng, Y. (2017): A new vector valued similarity measure for intuitionistic fuzzy sets based on OWA operators, Iranian Journal of Fuzzy Systems, accepted.

[15] Gong, Y., Su, X., Qian, H., Yang, N. (2017): Research on fault diagnosis methods for the reactor coolant system of nuclear power plant based on D-S evidence theory. Annals of Nuclear Energy, DOI: 10.1016/j.anucene.2017.10.026, 2017.

[16] Goyal, R. K., Kaushal, S. (2016): A constrained non-linear optimization model for fuzzy pairwise comparison matrices using teaching learning based optimization. Applied Intelligence, 1-10, 2016.

[17] Hu, Y., Du, F., Zhang, H. L., (2016): Investigation of unsteady aerodynamics effects in cycloidal rotor using RANS solver. Aeronauthical Journal 120 (122), 956-970, 2016.

[18] Inglis, J. (1977): A mathematical theory of evidence, Technometrics, 20 (1), 242, 1977.

[19] Jiang, W., Wang, S. (2017): An uncertainty measure for interval-valued evidences. International Journal of Computers Communications \& Control, 12 (5), 631-644, 2017.

[20] Jiang, W., Wang, S., Liu, X., Zheng, H., Wei, B. (2017): Evidence conflict measure based on OWA operator in open world. PloS ONE, 12 (5), e0177828, 2017.

[21] Jiang, W., Wei, B. (2018): Intuitionistic fuzzy evidential power aggregation operator and its application in multiple criteria decision-making. International Journal of Systems Science, 49 (3), 582-594, 2018.

[22] Jiang, W., Xie, C., Zhuang, M., Tang, Y. (2017): Failure mode and effects analysis based on a novel fuzzy evidential method, Applied Soft Computing, 57, 672-683. 
[23] Jiang, W., Zhan, J. (2017): A modified combination rule in generalized evidence theory. Applied Intelligence, 46 (3), 630-640, 2017.

[24] Jousselme, A. L., Grenier, D., loi Boss (2001): A new distance between two bodies of evidence. Information Fusion, 2 (2), 91-101, 2001.

[25] Kahraman, C., Onar, S. C., Oztaysi, B. (2015): Fuzzy multicriteria decision-making: A literature review. International Journal of Computational Intelligence Systems, 8 (4), 637$666,2015$.

[26] Kang, B., Chhipi-Shrestha, G., Deng, Y., Hewage, K., Sadiq, R. (2018): Stable strategies analysis based on the utility of Z-number in the evolutionary games. Applied Mathematics \& Computation, 324, 202-217, 2018.

[27] Kang, B., Chhipi-Shrestha, G., Deng, Y., Mori, J., Hewage, K., Sadiq, R. (2018): Development of a predictive model for Clostridium difficile infection incidence in hospitals using Gaussian mixture model and Dempster-Shafer theory, Stochastic Environmental Research and Risk Assessment, 32(6), 1743-1758, 2018.

[28] Kang, B., Deng, Y. (2018): Generating Z-number based on OWA weights usingmaximum entropy, International Journal of Intelligent Systems, https://doi.org/10.1002/int.21995, 2018.

[29] Li, C., Mahadevan, S. (2016): Relative contributions of aleatory and epistemic uncertainty sources in time series prediction, International Journal of Fatigue, 82, 474-486, 2016.

[30] Li, C., Mahadevan, S. (2016); Role of calibration, validation, and relevance in multi-level uncertainty integration. Reliability Engineering \& System Safety, 148, 32-43, 2016.

[31] Li, F., Zhang, X., Chen, X., Tian, Y. C. (2017): Adaptive and robust evidence theory with applications in prediction of floor water inrush in coal mine, Transactions of the Institute of Measurement \& Control, 39 (4), 2017.

[32] Li, Y., Chen, J., Ye, F., Liu, D., (2016). The Improvement of DS Evidence Theory and Its Application in IR/MMW Target Recognition, Journal of Sensors, (1903792), 2016.

[33] Liu, B., Hu, Y., Deng, Y. (2018): New failure mode and effects analysis based on d numbers downscaling method. International Journal of Computers Communications \& Control, 13 (2), 205-220, 2018.

[34] Liu, J., Lian, F., Mallick, M., (2016): Distributed compressed sensing based joint detection and tracking for multistatic radar system, Information Sciences, 369, 100-118, 2016.

[35] Liu, T., Deng, Y., Chan, F. (2017): Evidential supplier selection based on DEMATEL and game theory, International Journal of Fuzzy Systems, DOI: 10.1007/s40815-017-0400-4, 2017.

[36] Liu, W., Liu, H. B., Li, L. L. (2017): A multiple attribute group decision making method based on 2-d uncertain linguistic weighted heronian mean aggregation operator, International Journal of Computers Communications \& Control, 12(2):254-264, 2017.

[37] Mardani, A., Jusoh, A., Zavadskas, E. K. (2015): Fuzzy multiple criteria decision-making techniques and applications - two decades review from 1994 to 2014. Expert Systems with Applications, 42 (8), 4126-4148, 2015. 
[38] Meng, D., Zhang, H., Huang, T. (2016): A concurrent reliability optimization procedure in the earlier design phases of complex engineering systems under epistemic uncertainties, Advances in Mechanical Engineering, 8 (10), 2016.

[39] Mo, H., Deng, Y. (2016): A new aggregating operator in linguistic decision making based on D numbers. International Journal of Uncertainty, Fuzziness and Knowledge-Based Systems, 24 (6), 831-846, 2016.

[40] Pawlak, Z. (1982): Rough sets. International Journal of Computer \& Information Sciences, 11 (5), 341-356, 1982.

[41] Pedrycz, W., Al-Hmouz, R., Morfeq, A., Balamash, A. S. (2014): Building granular fuzzy decision support systems. Knowledge-Based Systems, 58, 3-10, 2014.

[42] Rekik, W., Hegarat-Mascle, S. L., Reynaud, R., Kallel, A. (2015): Dynamic estimation of the discernment frame in belief function theory, International Conference on Information Fusion, 1135-1142, 2015.

[43] Schubert, J. (2012): Constructing and evaluating alternative frames of discernment, Elsevier Science Inc., 2012.

[44] Shafer, G. (1976): Mathematical Theory of Evidence, Princeton University Press, Princeton, 1976.

[45] Smets, P. (1990): The combination of evidence in the transferable belief model. IEEE Transactions on Pattern Analysis \& Machine Intelligence, 12 (5), 447-458, 1990.

[46] Song, Y., Wang, X., Lei, L., Xing, Y., (2015): Credibility decay model in temporal evidence combination. Information Processing Letters, 115 (2), 248-252, 2015.

[47] Song, Y., Wang, X., Lei, L., Yue, S., (2016): Uncertainty measure for interval-valued belief structures, Measurement, 80, 241-250, 2016.

[48] Wang, J., Qiao, K., Zhang, Z., Xiang, F. (2017): A new conflict management method in dempster" cshafer theory. International Journal of Distributed Sensor Networks, 13, 3(20173-01) 13 (3), 155014771769650, 2017.

[49] Wen Jiang, Boya Wei, X. L. X. L., Zheng, H. (2017): Intuitionistic fuzzy power aggregation operator based on entropy and its application in decision making, International Journal of Intelligent Systems, https://doi.org/10.1002/int.21939, 2017.

[50] Xiao, F. (2016): An intelligent complex event processing with D numbers under fuzzy environment, Mathematical Problems in Engineering, 2016 (1), 1-10, 2016.

[51] Xiao, F. (2017): An improved method for combining conflicting evidences based on the similarity measure and belief function entropy. International Journal of Fuzzy Systems, DOI: $10.1007 / \mathrm{s} 40815-017-0436-5,2017$.

[52] Xiao, F. (2017): A novel evidence theory and fuzzy preference approach-based multi-sensor data fusion technique for fault diagnosis. Sensors, 17 (11), DOI: 10.3390/s17112504, 2017.

[53] Xiao, F., Aritsugi, M., Wang, Q., Zhang, R. (2016): Efficient processing of multiple nested event pattern queries over multi-dimensional event streams based on a triaxial hierarchical model. Artificial Intelligence in Medicine, 72 (C), 56-71, 2016. 
[54] Xiao, F., Zhan, C., Lai, H., Tao, L., Qu, Z. (2017): New parallel processing strategies in complex event processing systems with data streams. International Journal of Distributed Sensor Networks, 13 (8), 1-15, 2017.

[55] Xiao, F., Zhan, C., Lai, H., Tao, L., Qu, Z. (2017: New parallel processing strategies in complex event processing systems with data streams. International Journal of Distributed Sensor Networks, 13 (8), 1-15, 2017.

[56] Xu, H., Deng, Y. (2018): Dependent evidence combination based on shearman coefficient and pearson coefficient. IEEE Access, 6 (1), 11634-11640, 2018.

[57] Xu, S., Jiang, W., Deng, X., Shou, Y. (2017): A modified physarum-inspired model for the user equilibrium traffic assignment problem. Applied Mathematical Modelling, In Press, DOI: 10.1016/j.apm.2017.07.032, 2017.

[58] Yager, R. R. (2016): On viewing fuzzy measures as fuzzy subsets. IEEE Transactions on Fuzzy Systems, 24 (4), 811-818, 2016.

[59] Yager, R. R. (2016): Uncertainty modeling using fuzzy measures. Knowledge-Based Systems 92, 1-8, 2016.

[60] Yager, R. R., Elmore, P., Petry, F., 2017. Soft likelihood functions in combining evidence. Information Fusion, 36, 185-190.

[61] Ye, F., Chen, J., Li, Y., Kang, J., (2016). Decision-Making Algorithm for Multisensor Fusion Based on Grey Relation and DS Evidence Theory, Journal of Sensors, Article ID 3954573, http://dx.doi.org/10.1155/2016/3954573, 2016.

[62] Yin, L., Deng, Y. (2018): Measuring transferring similarity via local information. Physica A Statistical Mechanics \& Its Applications, 498, 102-115, 2018.

[63] Yuan, R., Meng, D., Li, H. (2016): Multidisciplinary reliability design optimization using an enhanced saddlepoint approximation in the framework of sequential optimization and reliability analysis. Journal of Risk \& Reliability, 230 (6), 2016.

[64] Zadeh, L. A. (2011): A note on Z-numbers, Information Sciences, 181 (14), 2923-2932, 2011.

[65] Zavadskas, E. K., Antuchevicience, J., Hajiagha, S. H. R. (2015): The interval-valued intuitionistic fuzzy multimoora method for group decision making in engineering, Mathematical Problems in Engineering, 560690, 2015.

[66] Zavadskas, E. K., Antucheviciene, J., Turskis, Z., Adeli, H., (2016): Hybrid multiplecriteria decision-making methods: A review of applications in engineering. Scientia Iranica, 23 (1), 1-20, 2016.

[67] Zhang, D., 2017. High-speed train control system big data analysis based on the fuzzy rdf model and uncertain reasoning. International Journal of Computers Communications \& Control, 12 (4), 577-591, 2017.

[68] Zhang, L., Wu, X., Qin, Y., Skibniewski, M. J., Liu, W. (2016). Towards a Fuzzy Bayesian Network Based Approach for Safety Risk Analysis of Tunnel-Induced Pipeline Damage. Risk Analysis, 36 (2), 278-301, 2016. 
[69] Zhang, L., Wu, X., Zhu, H., AbouRizk, S. M. (2017). Perceiving safety risk of buildings adjacent to tunneling excavation: An information fusion approach. Automation in Contruction, 73, 88-101, 2017.

[70] Zhang, Q., Li, M., Deng, Y. (2018): Measure the structure similarity of nodes in complex networks based on relative entropy. Physica A: Statistical Mechanics and its Applications, 491, 749-763, 2018.

[71] Zhang, R., Ashuri, B., Deng, Y. (2017): A novel method for forecasting time series based on fuzzy logic and visibility graph. Advances in Data Analysis and Classification, DOI: 10.1007/s11634-017-0300-3, 2017.

[72] Zhao, Y., Jia, R., Shi, P. (2016): A novel combination method for conflicting evidence based on inconsistent measurements. Information Sciences, 367-368, 125-142, 2016.

[73] Zheng, H., Deng, Y., (2018): Evaluation method based on fuzzy relations between Dempster-Shafer belief structure, International Journal of Intelligent Systems, https://doi.org/10.1002/int.21956, 2018.

[74] Zheng, X., Deng, Y. (2018): Dependence assessment in human reliability analysis based on evidence credibility decay model and iowa operator. Annals of Nuclear Energy, 112, 673-684, 2018.

[75] Zhou, X., Deng, X., Deng, Y., Mahadevan, S. (2017): Dependence assessment in human reliability analysis based on D numbers and AHP. Nuclear Engineering and Design, 313, 243-252, 2017.

[76] Zhou, X., Hu, Y., Deng, Y., Chan, F. T. S., Ishizaka, A. (2018): A DEMATEL - Based Completion Method for Incomplete Pairwise Comparison Matrix in AHP, Annals of Operations Research, https://doi.org/10.1007/s10479-018-2769-3, 2018. 